Original Research

\title{
Examining Muscle Activity Differences During Single and Dual Vector Elastic Resistance Exercises
}

\author{
Ryan WG Bench ${ }^{1}$, Sydney E Thompson ${ }^{1}$, Alan C Cudlip ${ }^{1}$, Michael WR Holmes, PhD ${ }^{1}$ a \\ ${ }^{1}$ Department of Kinesiology, Brock University \\ Keywords: biomechanics, electromyography, rehabilitation, theraband, upper limb \\ https://doi.org/10.26603/001c.21309
}

\section{International Journal of Sports Physical Therapy}

Vol. 16, Issue 2, 2021

\section{Background}

Elastic resistance exercise is a common part of rehabilitation programs. While these exercises are highly prevalent, little information exists on how adding an additional resistance vector with a different direction from the primary vector alters muscle activity of the upper extremity.

\section{Purpose}

The purpose of this study was to examine the effects of dual vector exercises on torso and upper extremity muscle activity in comparison to traditional single vector techniques.

\section{Study Design}

Repeated measures design.

\section{Methods}

Sixteen healthy university-aged males completed four common shoulder exercises against elastic resistance (abduction, flexion, internal rotation, external rotation) while using a single or dual elastic vector at a fixed cadence and standardized elastic elongation. Surface electromyography was collected from 16 muscles of the right upper extremity. Mean, peak and integrated activity were extracted from linear enveloped and normalized data and a 2-way repeated measures ANOVA examined differences between conditions.

\section{Results}

All independent variables differentially influenced activation. Interactions between single/dual vectors and exercise type affected mean activation in 11/16 muscles, while interactions in peak activation existed in $7 / 16$ muscles. Adding a secondary vector increased activation predominantly in flexion or abduction exercises; little changes existed when adding a second vector in internal and external rotation exercises. The dual vector exercise in abduction significantly increased mean activation in lower trapezius by $25.6 \pm 8.11 \% \mathrm{MVC}$ and peak activation in supraspinatus by $29.4 \pm 5.94 \% \mathrm{MVC}(\mathrm{p}<0.01)$. Interactions between single/dual vectors and exercise type affected integrated electromyography for most muscles; the majority of these muscles had the highest integrated electromyography in the dual vector abduction condition.

\section{Conclusion}

Muscle activity often increased with a second resistance vector added; however, the magnitude was exercise-dependent. The majority of these changes existed in the flexion and abduction exercises, with little differences in the internal or external rotation exercises.

\footnotetext{
a Corresponding author : 


\section{Level of Evidence}

$3 b$

\section{INTRODUCTION}

The shoulder complex is a mobile arrangement of joints and surrounding tissues which lends itself to a wide range of injury possibilities. The glenohumeral joint is one of the most mobile and inherently unstable joints in the human body due to its overall structure. ${ }^{1,2}$ With only surrounding musculature and the shallow glenoid fossa available to maintain control during motion, the glenohumeral joint is prone to injuries such as dislocations, impingement, subluxations, rotator cuff tears and tendonitis. ${ }^{2,3}$ Injuries to the shoulder complex account for approximately $10 \%$ of all athletic injuries. ${ }^{4}$ In particular, overhead sport athletes such as swimmers, tennis, volleyball and baseball players consistently place high tissue loads on the shoulder, and are often susceptible to injury. ${ }^{5}$ In order for an athlete or occupational worker to recover and return to work at peak performance, it is critical that the rehabilitation process target an optimized recovery plan such that the individual can return with a high level of strength and mobility.

Shoulder rehabilitation requires a multi-faceted approach. Rehabilitation programs can be complicated due to the need to balance the strength, flexibility and stability required on an individualized basis. ${ }^{6}$ One component of many shoulder rehabilitation programs is to use elastic resistance bands for resistance training, as they are inexpensive, versatile, adaptable and an effective way to incorporate external resistance. ${ }^{7-11}$ Elastic resistance has been demonstrated to promote increased shoulder strength, stability, and mobility. ${ }^{8,12}$ After training three times a week for six weeks using elastic resistance, collegiate baseball players showed a $20 \%$ increase in eccentric shoulder rotator muscle torque. ${ }^{13}$ Incorporating elastic resistance has also been demonstrated to increase shoulder muscle strength in competitive youth swimmers ${ }^{14}$ and elite-level tennis players, ${ }^{15}$ as well as increased throwing velocities in elite female handball players. ${ }^{16}$

Shoulder rehabilitation often focuses on increasing muscle activation of targeted musculature. The primary research in this area involves resistance exercises of singlejoint, uniplanar exercises that implement dumbbells or free weights to generate external resistance. ${ }^{17-20}$ However, a smaller number of studies have examined activation of shoulder musculature during rehabilitation using elastic bands and tubing, and these studies typically involve clinical populations or a small number of muscles. ${ }^{21-25}$ Using elastic bands can provide advantages in rehabilitation over free weights, as they provide increased control over phases of the movement, can act independent of gravity, and can easily modify resistance by adjusting the stretch. ${ }^{22}$ This elastic resistance provides similar muscle activation as isoinertial resistance, allowing relatively equal muscle adaptations between these two modes of exercise. ${ }^{26-28}$

Despite the prevalence and effectiveness of traditional elastic resistance rehabilitation and strengthening programs, little research has been conducted on dual vector exercises. Traditional work has focused on a single elastic resistance vector oriented to target the muscle of interest. This elastic generates loading through tension along the line of the elastic, and introducing a second elastic vector (dual vector) at a different location generates isotonic resistance in a direction other than gravity, altering the loading characteristics in rehabilitation programs. Dual vector exercises use additional lines of elastic loading to create forces in multiple directions. However, whether these modifications better target muscles of interest or alter activation beyond the primary muscle is unknown. The purpose of this study was to examine the effects of dual vector exercises on torso and upper extremity muscle activity in comparison to traditional single vector techniques. It was hypothesized that dual vector exercises would increase muscle activity compared to single vector exercises regardless of exercise selection.

\section{METHODS}

This study employed electromyography during elastic resistance exercises. Study design consisted of participants completing eight different testing conditions comprised of four single and four dual vector elastic resistance exercises on a single day, with each condition completed twice. Bipolar surface electrodes recorded muscular activation of 16 muscles surrounding the right torso and right upper extremity as participants completed shoulder rehabilitation exercises using single or dual vector resistance. Post-collection processing assessed changes in muscular activation across exercises and elastic resistance conditions.

\section{PARTICIPANTS}

Sixteen right-handed male subjects $[22.4 \pm 1.6$ years, $1.81 \pm$ $0.07 \mathrm{~m}, 82.1 \pm 8.6 \mathrm{~kg}$ ] participated after providing informed consent. Sample size was determined $a$ priori using $\mathrm{G}^{*}$ Power 3.1.9.7 (Universität Düsseldorf, Düsseldorf, Germany) and using effect sizes from previous elastic resistance literature. ${ }^{21,29}$ Exclusion criteria included shoulder, elbow, wrist or back pain or injury within the past 12 months. This study was approved by the institutional Research Ethics Board (BREB-17-346).

\section{INSTRUMENTATION}

Surface electromyography (EMG) was recorded from 16 right (dominant side) muscle sites (Figure 1): anterior, middle and posterior deltoid, clavicular and sternal heads of pectoralis major, serratus anterior, biceps, triceps, latissimus dorsi, flexor digitorum superficialis, extensor digitorum, supraspinatus, infraspinatus, and upper, middle and lower trapezius. After shaving the skin with a razor and cleansing with alcohol to minimize impedance, bipolar Ag$\mathrm{AgCl}$ surface electrodes (MediTrace 130, Kendall, Mansfield, MA, USA) were placed over the belly of each muscle based on published guidelines. ${ }^{30}$ A ground electrode was placed over the medial clavicle. EMG signals were sampled at 2160 
$\mathrm{Hz}$ and collected using a Bortec AMT-8 EMG system (Bortec Biomedical Ltd, Calgary, AB, Canada). Raw EMG signals were band-pass filtered from 10 to $1000 \mathrm{~Hz}$ and differentially amplified using a common-mode rejection ratio $>100$ $\mathrm{dB}$ with input impedance of $\sim 10 \mathrm{G} \Omega$.

\section{EXPERIMENTAL DESIGN}

The eight experimental conditions consisted of four different exercises (shoulder abduction, flexion, internal rotation, external rotation) completed using either single or dual vector elastic resistance. Elastic resistance was completed using Theraband ${ }^{\circledR}$ CLX (Performance Health, Akron, OH, USA). Theraband ${ }^{\circledR}$ CLX consists of elastic bands in fused links throughout the length of the elastic, allowing loops at various lengths as well as multiple connection points to the body within a single band.

\section{PROTOCOL}

The protocol included application of surface electrodes, collection of maximum voluntary contractions (MVCs), a training period and experimental trials. Following electrode placement, participants completed five second muscle specific MVCs resisted by a researcher who had extensive experience with generating effective MVCs, with each muscle completed twice. ${ }^{30} \mathrm{~A}$ minimum of one minute of rest was provided between contractions and participants received verbal encouragement during MVCs. ${ }^{31,32}$ Following MVCs, all experimental conditions were explained verbally and demonstrated by the researcher, then participants had time to practice each condition to ensure proper technique. Participants self-selected elastic resistance level; participants were instructed to choose a resistance level that would allow them to complete 12-15 continuous repetitions without fatigue. ${ }^{33}$ All participants chose either the green or blue elastic resistance bands ( 2.1 and $2.6 \mathrm{~kg}$ at $100 \%$ elongation, respectively), which are intermediate band colors and appropriate resistances for shoulder exercises. ${ }^{22}$ All exercises for a participant were done with the same color bands at the same length, and each exercise began with the band at $125 \%$ elongation. ${ }^{29}$

Four elastic rehabilitation exercises were evaluated (Figure 2 and $\underline{3}$ ). Participants completed these exercises using their right arm. These exercises included: 1) shoulder abduction - arm motion from $0^{\circ}$ to maximal elevation in the coronal (abduction) plane with the resistance anchored between the foot and hand, 2) flexion - arm motion from $0^{\circ}$ to maximal elevation in the sagittal plane with the resistance anchored between the foot and hand, 3) internal and 4) external rotation, where internal and external rotation required the arm in the frontal plane and the humerus at $90^{\circ}$ of arm elevation with the elbow in $90^{\circ}$ of flexion; ${ }^{20}$ resistance was anchored to a fixed surface in front of the participant at shoulder height. Each exercise required the participants to hold the elastic resistance band in their right hand. The dual vector was placed such that this vector was parallel to the ground at the height of the elbow in internal and external rotation tasks, and in line with the mediolateral axis of the elbow in flexion and abduction tasks. Participants were instructed to keep their elbow straight during
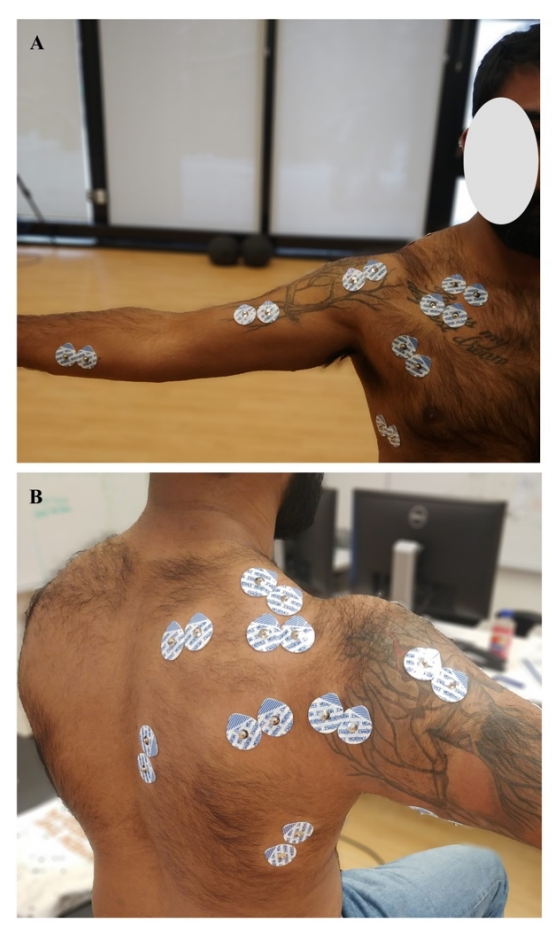

Figure 1: Experimental set up

flexion and abduction trials, and to maintain a $90^{\circ}$ elbow angle in the external rotation trials. Motion was visually inspected during trials by two researchers in different planes (front, right side) to ensure proper technique. If a participant did not maintain the required technique during collection the participant was provided feedback and the trial was recollected.

Internal and external rotation dual vector exercises were completed with one elastic resistance band held in the hand, and an additional elastic band looped over the elbow. The elastic resistance was mounted to a fixed surface such that the vector at the elbow was parallel with the floor at approximately shoulder height (Figure 3B, 3D). Abduction and flexion dual vector exercises had two bands held in the hand while the second vector added resistance in a perpendicular plane to the motion (Figure 2B, 2D). In each exercise, participants completed three repetitions at a fixed cadence of two seconds per repetition, one second concentric, one second eccentric as informed by a metronome at $60 \mathrm{bpm}$. Participants completed two sets for all conditions in a fully randomized order, with a minimum of one minute of rest between exercises.

\section{DATA ANALYSIS}

EMG was analyzed with respect to amplitude. MVC and experimental data were processed identically. Raw EMG signals were high-pass filtered at $30 \mathrm{~Hz}$ to eliminate ECG contamination. ${ }^{34}$ These data were then full-wave rectified and linear enveloped using a dual-pass fourth-order low-pass Butterworth filter at $4 \mathrm{~Hz}$. Mean, peak and integrated EMG via trapezoidal integration (iEMG) was calculated for each muscle from each set of exercises and normalized to the single highest peak of the two muscle-specific MVC trials. 


\section{STATISTICAL ANALYSIS}

Statistical analysis was focused on muscle activation changes from differing exercises and the use of single or dual elastic resistance. Dependent variables included mean, peak and integrated EMG for each of the 16 muscles in all eight conditions (four exercises, two elastic resistance settings). Prior to statistical analysis, a Shapiro-Wilk test was completed for each dependent variable to examine normality; in all cases, the null hypothesis was retained. A 2-way repeated measures ANOVA with 2 independent factors (exercise, number of elastic resistance vectors) with one 2-way interaction examined muscle activity changes. Statistical significant was set at $\alpha=0.05$. Tukey HSD post-hoc and t-tests were performed to examine statistically significant main effects and interactions. All statistical analyses were completed using JMP 14.0 (SAS Institute, North Carolina, USA).

\section{RESULTS}

All independent variables differentially influenced mean, peak, and integrated muscle activation as interactions or main effects. This results section has been subdivided by dependent variable.

\section{MEAN ACTIVATION}

Interactions between exercise and single/dual resistance vectors affected mean EMG for most muscles, while those that did not display interactions demonstrated main effects. Interactions existed in the anterior, middle and posterior deltoid, sternal and clavicular heads of pectoralis major, triceps, supraspinatus, infraspinatus, and upper, middle and lower trapezius ( $p<0.001$ to 0.022 ) (Table 1 ). The dual vector abduction exercise generated the highest mean EMG in middle deltoid, posterior deltoid, triceps, supraspinatus, infraspinatus, upper trapezius, middle trapezius, and lower trapezius, with mean values ranging from 9.41-77.6 \%MVC, while the dual vector flexion exercise generated the highest mean EMG in the anterior deltoid and latissimus dorsi at $25.78 \pm 1.37$ and $14.22 \pm 1.74 \%$ MVC, respectively (Table 1 ). Activation across muscles was typically highest in the flexion or abduction exercises, and internal rotation typically had the lowest activation (Figure 4A). Single and dual vectors altered activation in nine muscles during abduction, and six muscles during flexion (Figure 4B). Main effects of exercise affected the biceps, flexor digitorum, extensor digitorum and serratus anterior $(\mathrm{p}<0.001)$. Mean activation in the biceps and serratus anterior was higher during the abduction and flexion exercises than the internal or external rotation exercises, but these two exercises were not significantly different from one another. Mean activation in the flexor digitorum during the internal rotation exercise (11.83 \pm 2.88 \%MVC) were higher than the external rotation or flexion trials (9.37 and $9.38 \% \mathrm{MVC}$, respectively), but no other exercises were different from one another. Mean activation in the forearm extensors were highest in the internal rotation exercise $(16.1 \pm 3.17 \% \mathrm{MVC})$ were higher than the flexion trial (11.29\%MVC), but no other post-hoc differences between exercises were present. Main effects of sin-
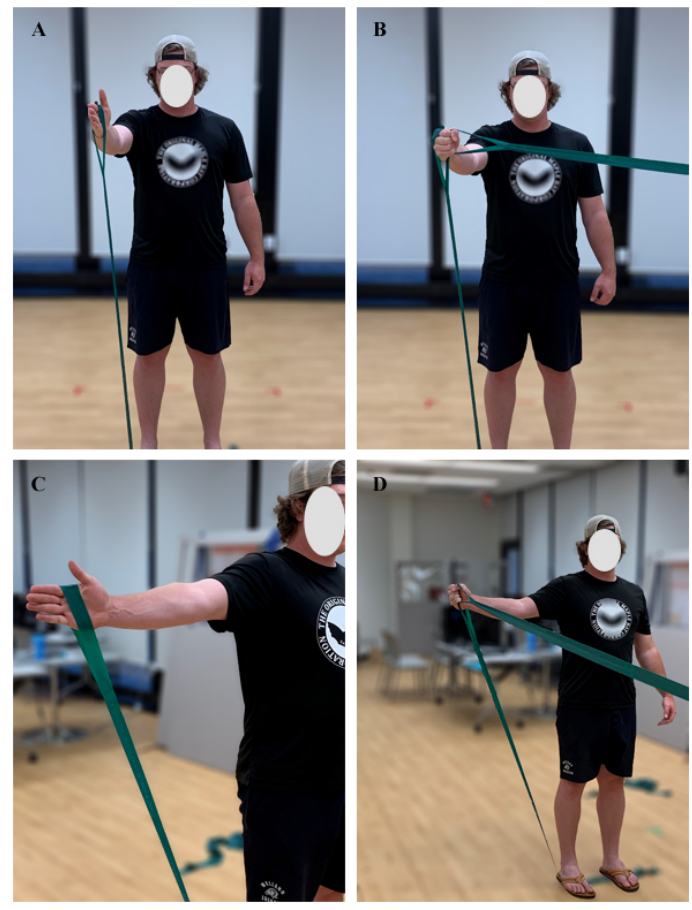

Figure 2: Exercise conditions, single and dual vector. $A=$ flexion, single vector, $B=$ flexion, dual vector, $C=$ abduction, single vector, $D=$ abduction, dual vector
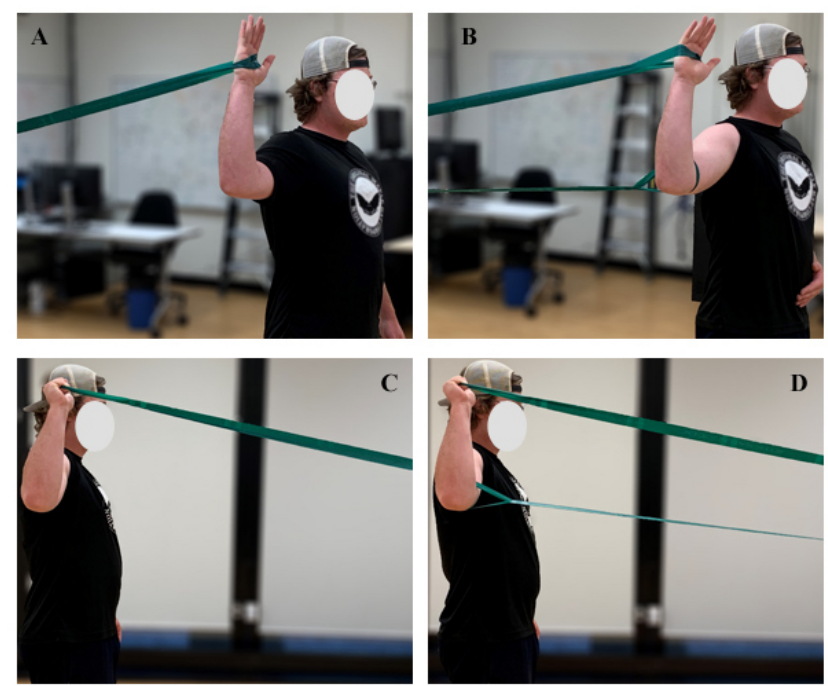

Figure 3: Exercise conditions, single and dual vector, continued. $A=$ internal rotation, single vector, $B=$ internal rotation, dual vector, $\mathrm{C}=$ external rotation, single vector, $D=$ external rotation, dual vector

gle/dual vector exercises were present in the flexor digitorum and extensor digitorum $(\mathrm{p}<0.001)$; in both muscles, activation was higher in the dual vector exercise.

\section{PEAK ACTIVATION}

Interactions between exercise and single/dual vector af- 
fected peak EMG for some muscles; those with no interaction had significant main effects of exercise. Interactions were present in the middle and posterior deltoid, supraspinatus, infraspinatus, and upper, middle and lower trapezius ( $\mathrm{p}=0.0005-0.0437$ ) (Table 2). The dual vector $\mathrm{ab}$ duction exercise had the highest activation for all of these exercises except for the infraspinatus, with the highest activation during the dual vector external rotation exercise (86.62 $\pm 6.32 \% \mathrm{MVC})$. Within an exercise, moving from single to dual vectors increased activation in abduction for six muscles (Figure 5). Main effects of exercise were observed in the anterior deltoid, sternal and clavicular heads of pectoralis major, serratus anterior, biceps, triceps, latissimus dorsi, and extensor digitorum ( $\mathrm{p}<0.001-0.004)$. Across all of these muscles, the peak EMG in internal rotation was significantly lower than other exercises and significantly higher in flexion or abduction except for extensor digitorum, where internal rotation $(40.46 \pm 10.16 \% \mathrm{MVC})$ was significantly greater than flexion $(24.68 \pm 7.96 \% \mathrm{MVC})$, but no other exercises were different from one another (Figure 5A). A main effect of single/dual vector affected peak EMG in the middle deltoid $(\mathrm{p}=0.0088)$ and latissimus dorsi $(\mathrm{p}=$ 0.0324); use of a dual vector increased peak EMG in both of these muscles from 48.07 to $52.78 \pm 4.23 \%$ MVC and 30.53 to $35.21 \pm 6.46 \%$ in middle deltoid and latissimus dorsi, respectively (Figure 5B). No significant changes were present for flexor digitorum.

\section{INTEGRATED EMG}

Interactions between exercise and single/dual vectors influenced iEMG for most muscles; all other muscles were affected by main effects of exercise selection or number of resistance vectors. Interactions affected integrated EMG of the anterior, middle and posterior deltoid, sternal and clavicular heads of pectoralis major, triceps, latissimus dorsi, supraspinatus, infraspinatus, and upper, middle and lower trapezius ( $\mathrm{p}<0.001-0.0198$ ) (Table 3). All of these muscles except for anterior deltoid, the clavicular head of pectoralis major and latissimus dorsi had the highest integrated EMG in the dual vector condition during abduction. Peak integrated EMG in the anterior deltoid and clavicular head of pectoralis major was highest in the single vector flexion exercise (275145.2 \pm 17799.8 and $102106.6 \pm 7336.0 \%$ MVC* $s$, respectively). Latissimus dorsi integrated activation was highest in the dual vector condition during flexion $(182728.0 \pm 22557.8 \%$ MVC*s). Main effects of exercise were present in serratus anterior, biceps, flexor digitorum and extensor digitorum $(\mathrm{p}<0.001-0.395)$. In serratus anterior and biceps, the abduction and flexion conditions increased integrated activation compared to internal or external rotation by up to $163 \%$ and $162 \%$ respectively, but no other differences were present. The internal rotation condition generated $42 \%$ higher integrated activation compared to the flexion condition in extensor digitorum and $27 \%$ higher than flexion or external rotation conditions in flexor digitorum, but no other differences were present.

\section{DISCUSSION}

The purpose of this study was to investigate differences in
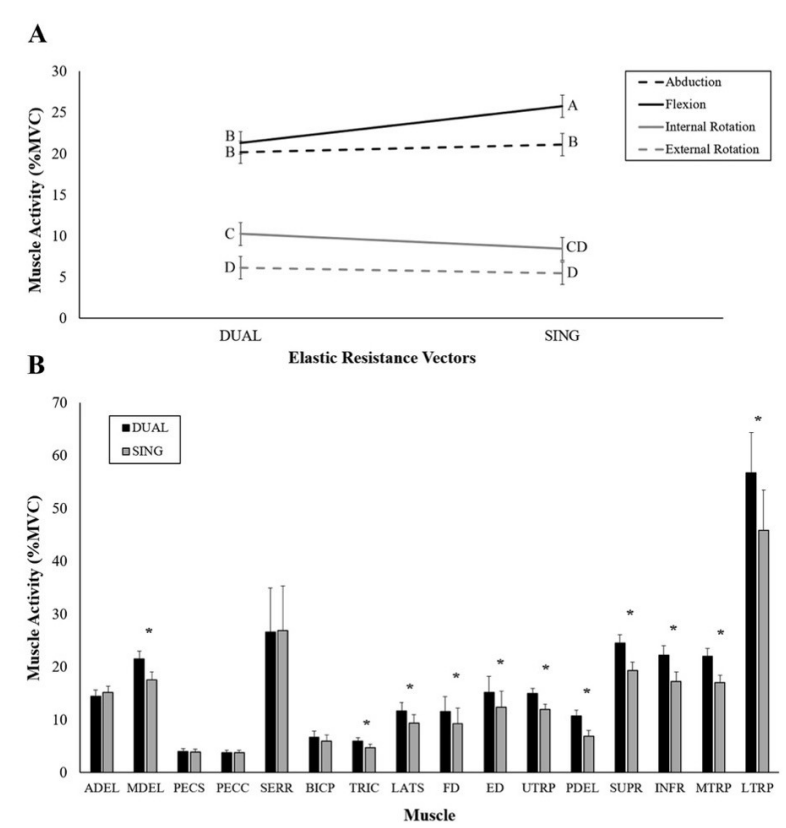

Figure 4: A) An interaction between exercise and number of elastic vectors affected mean activation in anterior deltoid, with increased activation in the single vector flexion task. Significant differences in this interaction are denoted by letters; data points not sharing a letter are significantly different. B) Changing between single and dual vectors in flexion altered activation in eleven muscles; differences between single and dual vector activation is indicated by asterisks.

muscle activation between dual vector and traditional single vector resistance exercises. The location and direction of the secondary vector in the abduction and flexion exercises created a resistance beyond the primary vector. In the internal and external rotation conditions, the secondary vector resisted horizontal adduction and abduction, respectively. The hypothesis of this work was that the dual vector exercise would increase muscle activation was partially supported, with increased activation for some muscles in the abduction and flexion dual vector exercises, but minimal changes existed when adding a dual vector to the internal or external rotation tasks, indicating that adding a dual vector was not universally beneficial.

Although the abduction and flexion dual vector exercises were effective at increasing global activation, the abduction task increased activity in a greater number of muscles; changing to dual vector exercises with internal or external rotation appeared to result in only marginal changes to muscle activation. The dual vector exercise condition for abduction increased mean activation in nine muscles, peak activation in six muscles and iEMG in nine muscles, while the dual vector flexion exercise increased mean activation in six muscles, no muscles in peak activation, and three muscles in iEMG. Horizontal abduction and elevation in the frontal plane has been demonstrated to target the upper and lower trapezius more than flexion exercises. ${ }^{17}$ In the 
abduction task, the primary vector provided resistance to elevation in the frontal plane, while the dual vector resisted horizontal abduction. As both of these movements target the trapezius, this may explain differences between the flexion and abduction tasks. As the posterior deltoid is a horizontal abductor at the shoulder, changes in activity were expected with the addition of the dual vector; however, changes were only observed in the abduction and external rotation conditions. Maximum EMG activity is expected from the posterior deltoid during horizontal abduction at $90^{\circ}$ abduction, ${ }^{35}$ a similar posture was adopted during the abduction and external rotation dual vector conditions. Supraspinatus activity only increased in the abduction dual vector condition; the supraspinatus operates as a shoulder abductor and glenohumeral stabilizer and is expected to have greatest activity in abduction or horizontal abduction. ${ }^{36,37}$ Adding a second vector to internal or external rotation tasks did not appear to alter muscle activation across the muscles examined. The dual vector exercises only significantly increased muscle activation in the mean and iEMG values for the clavicular head of pectoralis major in internal rotation and the posterior deltoid in external rotation. In both of these cases, the difference in mean activation was less than 4.1 \%MVC. While these increases in activation may be statistically significant, the small absolute difference may not identify these changes as clinically meaningful. ${ }^{38,39}$

Shoulder rehabilitation exercises are designed to isolate specific muscles while remaining cognizant of potential subacromial space changes. During active arm elevation, particularly in flexion and abduction, the rotator cuff and scapular muscles play an important role in maintaining the subacromial space. ${ }^{40-43}$ With the addition of the dual vector in abduction, increased activity was seen in the middle and posterior deltoids. This would likely joint compression at $90^{\circ}$ of abduction, as this compressive force is centralized in the glenoid fossa. ${ }^{37}$ Increasing co-contraction of adductor muscles has been demonstrated to reduce patient complaints and pain scores in individuals with subacromial pain syndrome; ${ }^{44}$ increasing activation in rehabilitation could lead to beneficial outcomes in the future. Exercises or movements that protect against SLAP lesions are ones that activate biceps, triceps, latissimus dorsi and upper trapezius ${ }^{45}$ adding a second vector increased mean activation in triceps, latissimus dorsi and upper trapezius, which may be beneficial for shoulder health. If the goal of dual vector exercises is to increase global activation, this method of exercise may be suitable for use towards the end of a rehabilitation program as a progression of traditional resistance band exercises. Recent research has incorporated this dual vector rehabilitation strategy for overhead athletes ${ }^{46}$ in an attempt to modulate lower trapezius activation, and found increased activation when using a dual vector strategy compared to a single vector. As the lower trapezius maintains scapular position during the late cocking phase of throwing, ${ }^{47}$ it is plausible that this dual vector strategy would alleviate lower trapezius activation, which is associated with scapular dyskinesis and SLAP lesions in baseball pitchers. ${ }^{48}$ This work observed increased mean, peak and integrated lower trapezius activation in dual vector exercises, supporting these findings.
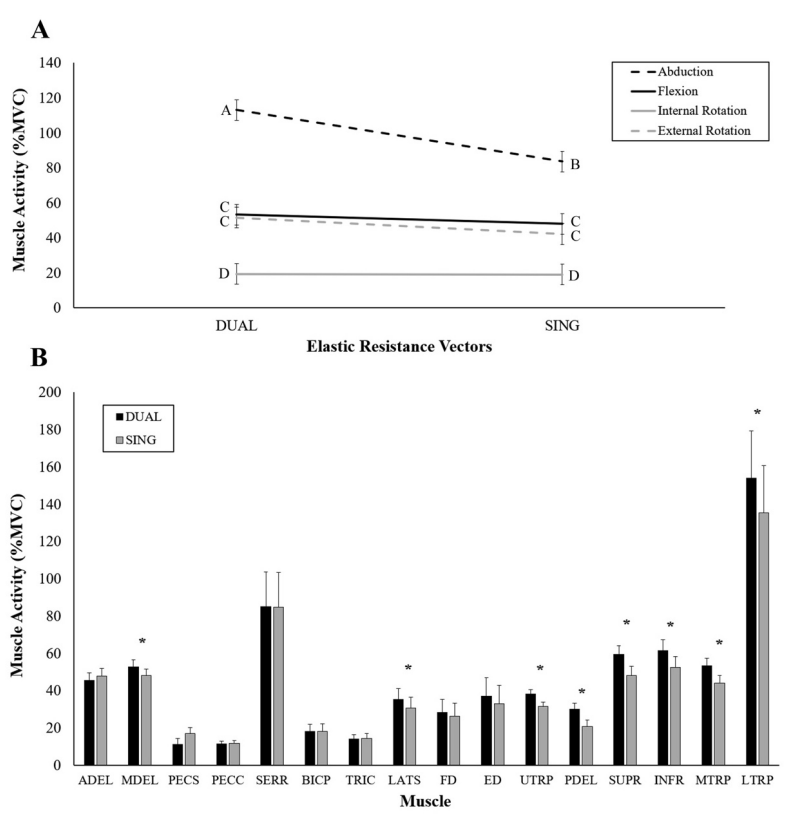

Figure 5: A) An interaction between exercise and number of elastic vectors affected peak activation in supraspinatus, with increased activation in the dual vector abduction task. Significant differences in this interaction are denoted by letters; data points not sharing a letter are significantly different. B) Changing between single and dual vectors in flexion altered activation in eight muscles; differences between single and dual vector activation is indicated by asterisks.

Some inherent limitations are present in this work. The population studied was limited to healthy university aged males who had no shoulder or arm pain in the prior 12 months, limiting its potential utility for older or pathologic populations. Only four exercises were investigated, however many shoulder exercises using elastic resistance bands exist for different shoulder rehabilitation purposes. The exercises selected are commonly used and represent a range of elastic resistance postures. The second vector was held in the hand for abduction and flexion, versus being attached at the elbow for internal and external rotation. This might have caused the resistance arm of the dual vector to be smaller, potentially decreasing the second vector's load at the shoulder. The concentric and eccentric phases were not divided within a repetition for dependent variable calculation; as such, it is difficult to comment on timing of these activations. Future research should differentiate between concentric and eccentric phases of these motions to expand on these findings. RPE was not collected during experimental trials; while participants were instructed to select a band that would allow for 12-15 continuous repetitions, there may be variations in intensity across participants. Due to varying resistance arm lengths with different band attachment locations, variable band tensions should be investigated further, as this study was done with low resistance. However, band lengths were normalized to $125 \%$ stretch at the peak of each repetition in each exercise. 
Table 1: Exercise * Single/Dual vector interactions for normalized mean muscle activity (\%MVC).

\begin{tabular}{|c|c|c|c|c|c|c|c|c|c|c|c|}
\hline \multirow[b]{2}{*}{ Muscle } & \multirow{2}{*}{ p value } & \multirow{2}{*}{ F Ratio } & \multirow{2}{*}{ Effect Size $\left(\eta_{\mathrm{P}}{ }^{2}\right)$} & \multicolumn{2}{|c|}{ Abduction } & \multicolumn{2}{|c|}{ External Rotation } & \multicolumn{2}{|c|}{ Flexion } & \multicolumn{2}{|c|}{ Internal Rotation } \\
\hline & & & & Single & Dual & Single & Dual & Single & Dual & Single & Dual \\
\hline ADEL & 0.0012 & 5.47 & 0.79 & $21.11_{B}$ & $20.17_{B}$ & ${ }^{5.47 D}$ & 6.12D & $21.29_{B}$ & $25.78_{A}$ & $10.22 \mathrm{C}$ & ${ }^{8.42} \mathrm{CD}$ \\
\hline MDEL & $<0.0001$ & 7.47 & 0.82 & $28.75_{B}$ & $37.33_{\mathrm{A}}$ & $15.26 \mathrm{D}$ & $18.73_{D}$ & $15.26 \mathrm{CD}$ & $19.54 \mathrm{CD}$ & $9.68_{E}$ & $10.18_{\mathrm{E}}$ \\
\hline PDEL & $<0.0001$ & 12.22 & 0.73 & ${ }^{10.06} \mathrm{C}$ & $18.74_{A}$ & ${ }^{10.48} \mathrm{C}$ & $14.50_{B}$ & $3.78_{\mathrm{DE}}$ & $6.60_{D}$ & $3.25_{E}$ & $2.97_{E}$ \\
\hline PECS & 0.0224 & 3.25 & 0.76 & ${ }^{4.14} \mathrm{AB}$ & $4.11_{\mathrm{AB}}$ & $3.10_{C}$ & $3.33_{\mathrm{BC}}$ & $4.96_{\mathrm{AB}}$ & $4.42 \mathrm{AB}$ & $3.37_{\mathrm{BC}}$ & $4.18_{A B}$ \\
\hline PECC & $<0.0001$ & 12.40 & 0.60 & ${ }^{2.86} \mathrm{CDE}$ & $3.46 \mathrm{CD}$ & $1.78_{\mathrm{E}}$ & ${ }^{1.94} \mathrm{DE}$ & ${ }^{7.87} \mathrm{~A}$ & $5.14_{B}$ & $2.70_{\mathrm{DE}}$ & $4.42_{\mathrm{BC}}$ \\
\hline SERR & 0.9798 & 0.06 & 0.66 & 33.48 & 32.08 & 20.42 & 19.29 & 39.74 & 38.79 & 14.04 & 15.94 \\
\hline $\mathrm{BICP}$ & 0.3439 & 0.79 & 0.70 & 8.16 & 9.47 & 4.39 & 4.60 & 8.38 & 8.65 & 2.96 & 3.82 \\
\hline TRIC & $<0.0001$ & 10.33 & 0.77 & $6.35_{\mathrm{BC}}$ & $9.41_{A}$ & $4.26_{\mathrm{DE}}$ & 4.45 $\mathrm{DE}$ & $5.02 \mathrm{CD}$ & $6.84_{B}$ & $3.33_{E}$ & $3.03_{E}$ \\
\hline LATS & 0.0002 & 6.97 & 0.82 & $8.93_{D}$ & ${ }^{13.79} \mathrm{AB}$ & $11.19_{\mathrm{BCD}}$ & ${ }^{12.72} \mathrm{ABC}$ & ${ }^{10.92} \mathrm{CD}$ & $14.22 \mathrm{~A}$ & $6.24 \mathrm{E}$ & $5.77_{\mathrm{E}}$ \\
\hline FDS & 0.3181 & 1.17 & 0.88 & 9.53 & 12.55 & 8.70 & 10.04 & 7.72 & 11.04 & 11.27 & 12.38 \\
\hline ED & 0.4748 & 0.83 & 0.63 & 11.00 & 16.20 & 13.44 & 14.95 & 9.32 & 13.27 & 15.81 & 16.39 \\
\hline SUPR & $<0.0001$ & 8.52 & 0.78 & $32.43_{B}$ & $44.64_{\mathrm{A}}$ & $19.18_{C D}$ & ${ }^{23.65} \mathrm{C}$ & ${ }^{16.1_{D}}$ & $19.97 \mathrm{CD}$ & $9.34_{\mathrm{E}}$ & $9.58_{E}$ \\
\hline INFR & $<0.0001$ & 13.21 & 0.81 & ${ }^{18.22} \mathrm{C}$ & $28.33_{\mathrm{A}}$ & $23.73_{B}$ & $27.11_{A B}$ & ${ }^{16.99} \mathrm{C}$ & $23.88_{B}$ & $9.81_{D}$ & $9.50_{D}$ \\
\hline UTRP & $<0.0001$ & 11.02 & 0.83 & $19.01_{B}$ & $26.3_{A}$ & ${ }^{8.62} \mathrm{DE}$ & $10.96 \mathrm{D}$ & ${ }^{14.94} \mathrm{C}$ & $16.58_{\mathrm{BC}}$ & $5.38_{F}$ & $5.86_{\mathrm{EF}}$ \\
\hline MTRP & $<0.0001$ & 13.45 & 0.82 & $26.99_{B}$ & $38.49 \mathrm{~A}$ & $20.25 \mathrm{CD}$ & $24.17_{\mathrm{BC}}$ & $12.32_{\mathrm{E}}$ & $17.65_{D}$ & $8.44_{F}$ & ${ }^{7.91} 1_{\mathrm{EF}}$ \\
\hline LTRP & $<0.0001$ & 9.87 & 0.81 & $52.06_{\mathrm{BC}}$ & $77.6_{A}$ & $65.37 \mathrm{AB}$ & ${ }^{75.76} \mathrm{~A}$ & ${ }^{41.2} \mathrm{C}$ & $56.72_{\mathrm{BC}}$ & $24.74 \mathrm{D}$ & ${ }^{16.64} \mathrm{D}$ \\
\hline
\end{tabular}

Note: Muscles with significant interactions have their rows highlighted grey; post-hoc differences within that muscle are denoted by letters within data cells; values in a row not sharing a letter are significantly different from one another. $\mathrm{ADEL}=$ Anterior deltoid; $\mathrm{MDEL}=$ Middle deltoid; $\mathrm{PDEL}=$ Posterior deltoid; $\mathrm{PECS}=$ Pectoralis major (sternal head);

PECC $=$ Pectoralis major $($ clavicular head $) ;$ SERR $=$ Serratus anterior; BICP $=$ Biceps; TRIC $=$ Triceps; LATS $=$ Latissimus dorsi;

FDS = Flexor digitorum superficialis; ED = Extensor digitorum; SUPR $=$ Supraspinatus; INFR = Infraspinatus;

UTRP = Upper trapezius; MTRP = Middle trapezius; LTRP = Lower trapezius 
Table 2: Exercise * Single/Dual vector interactions for normalized peak muscle activity (\%MVC).

\begin{tabular}{|c|c|c|c|c|c|c|c|c|c|c|c|}
\hline \multirow[b]{2}{*}{ Muscle } & \multirow{2}{*}{$p$ value } & \multirow{2}{*}{ F Ratio } & \multirow{2}{*}{ Effect Size $\left(n_{P}{ }^{2}\right)$} & \multicolumn{2}{|c|}{ Abduction } & \multicolumn{2}{|c|}{ External Rotation } & \multicolumn{2}{|c|}{ Flexion } & \multicolumn{2}{|c|}{ Internal Rotation } \\
\hline & & & & Single & Dual & Single & Dual & Single & Dual & Single & Dual \\
\hline ADEL & 0.0799 & 2.28 & 0.78 & 75.70 & 73.82 & 12.79 & 14.02 & 81.87 & 70.00 & 20.61 & 24.39 \\
\hline MDEL & 0.0437 & 2.85 & 0.79 & $79.13_{B}$ & $91.87_{A}$ & $31.89 \mathrm{D}$ & $36.78_{D}$ & $54.86_{C}$ & ${ }^{55.70_{C}}$ & $26.40_{D}$ & $26.75 \mathrm{D}$ \\
\hline PDEL & 0.0026 & 4.88 & 0.65 & $28.96 \mathrm{CD}$ & $48.51_{\mathrm{A}}$ & $33.16_{\mathrm{BC}}$ & ${ }^{42.61_{A B}}$ & $13.33_{\mathrm{EF}}$ & $20.22 \mathrm{DE}$ & $8.13_{F}$ & $8.57_{F}$ \\
\hline PECS & 0.096 & 2.13 & 0.17 & 16.40 & 14.99 & 7.13 & 7.39 & 15.43 & 10.56 & 8.67 & 12.29 \\
\hline PECC & 0.487 & 0.81 & 0.39 & 10.40 & 11.13 & 4.75 & 4.85 & 22.41 & 19.05 & 9.25 & 11.19 \\
\hline SERR & 0.8872 & 0.21 & 0.60 & 122.50 & 132.12 & 72.16 & 66.44 & 115.87 & 109.29 & 28.65 & 32.52 \\
\hline $\mathrm{BICP}$ & 0.6839 & 0.49 & 0.63 & 27.47 & 28.49 & 12.36 & 11.6 & 26.45 & 23.24 & 6.60 & 8.91 \\
\hline TRIC & 0.569 & 0.67 & 0.19 & 16.32 & 21.72 & 11.60 & 10.45 & 22.5 & 17.71 & 7.50 & 6.50 \\
\hline LATS & 0.0775 & 2.30 & 0.69 & 29.25 & 42.19 & 40.13 & 45.71 & 36.05 & 39.32 & 16.67 & 13.62 \\
\hline FDS & 0.7577 & 0.39 & 0.55 & 33.42 & 31.05 & 23.58 & 30.80 & 21.89 & 23.63 & 26.16 & 27.51 \\
\hline ED & 0.6864 & 0.49 & 0.59 & 30.12 & 35.71 & 36.73 & 47.55 & 23.28 & 26.08 & 41.92 & 39.0 \\
\hline SUPR & 0.0027 & 4.84 & 0.70 & $83.70_{B}$ & $113.11_{A}$ & ${ }^{42.23} \mathrm{C}$ & ${ }^{51.59} \mathrm{C}$ & ${ }^{47.97} \mathrm{C}$ & ${ }^{53.35_{C}}$ & $19.06_{\mathrm{D}}$ & $19.44_{D}$ \\
\hline INFR & 0.0002 & 6.87 & 0.77 & ${ }^{46.78}{ }_{C D}$ & $69.05_{B}$ & $83.14 \mathrm{~A}$ & $86.62 \mathrm{~A}$ & $45.93 \mathrm{D}$ & $58.92_{\mathrm{BC}}$ & 34.52 $\mathrm{DE}$ & $31.51_{\mathrm{E}}$ \\
\hline UTRP & 0.0005 & 6.09 & 0.79 & $53.54_{B}$ & $70.78_{A}$ & ${ }^{18.81_{\mathrm{DE}}}$ & $23.76 \mathrm{D}$ & ${ }^{42.60_{C}}$ & $45.98_{\mathrm{BC}}$ & $11.47_{\mathrm{E}}$ & $12.44_{\mathrm{E}}$ \\
\hline MTRP & 0.0041 & 4.54 & 0.74 & $69.64_{B}$ & $91.43_{\mathrm{A}}$ & $49.69_{C D}$ & $57.77_{\mathrm{BC}}$ & $38.16 \mathrm{D}$ & $45.29 \mathrm{CD}$ & $18.05_{\mathrm{E}}$ & $18.43_{\mathrm{E}}$ \\
\hline LTRP & 0.0209 & 3.30 & 0.79 & $149.76_{\mathrm{BC}}$ & $201.16 \mathrm{~A}$ & $194.46_{\mathrm{AB}}$ & $215.24_{A}$ & ${ }^{130.41_{C}}$ & ${ }^{147.01_{C}}$ & $66.90_{D}$ & $52.80_{\mathrm{D}}$ \\
\hline
\end{tabular}

Note: Muscles with significant interactions have their rows highlighted grey; post-hoc differences within that muscle are denoted by letters within data cells; values in a row not sharing a letter are significantly different from one another. $\mathrm{ADEL}=$ Anterior deltoid; $\mathrm{MDEL}=$ Middle deltoid $; \mathrm{PDEL}=$ Posterior deltoid; $\mathrm{PECS}=$ Pectoralis major (sternal head);

PECC $=$ Pectoralis major $($ clavicular head $) ;$ SERR $=$ Serratus anterior; BICP $=$ Biceps; TRIC $=$ Triceps; LATS $=$ Latissimus dorsi;

FDS = Flexor digitorum superficialis; ED = Extensor digitorum; SUPR $=$ Supraspinatus; INFR = Infraspinatus;

UTRP = Upper trapezius; MTRP = Middle trapezius; LTRP = Lower trapezius 
Table 3: Exercise * Single/Dual vector interactions for normalized integrated muscle activity (\%MVC"s).

\begin{tabular}{|c|c|c|c|c|c|c|c|c|c|c|c|}
\hline \multirow[b]{2}{*}{ Muscle } & \multirow{2}{*}{$p$ value } & \multirow{2}{*}{$\begin{array}{c}F \\
\text { Ratio }\end{array}$} & \multirow{2}{*}{$\begin{array}{l}\text { Effect } \\
\text { Size ( } \\
\left.\eta_{p}{ }^{2}\right) \\
\end{array}$} & \multicolumn{2}{|c|}{ Abduction } & \multicolumn{2}{|c|}{ External Rotation } & \multicolumn{2}{|c|}{ Flexion } & \multicolumn{2}{|c|}{ Internal Rotation } \\
\hline & & & & Single & Dual & Single & Dual & Single & Dual & Single & Dual \\
\hline ADEL & 0.0010 & 5.56 & 0.79 & $273687.5_{B}$ & $261437.5_{B}$ & 79394.4D & $70927.0_{D}$ & 334218.7 A & $275145.2_{B}$ & $108234.1_{C D}$ & ${ }^{131921.3 C}$ \\
\hline MDEL & 0.0001 & 7.29 & 0.82 & $372750.0_{B}$ & $483812.5_{\mathrm{A}}$ & $197874.8 \mathrm{D}$ & $242714.9 \mathrm{CD}$ & $214642.4_{C D}$ & $252588.63_{C D}$ & $123809.0_{E}$ & $131210.0_{\mathrm{E}}$ \\
\hline PDEL & $<0.0001$ & 12.21 & 0.73 & $130378.0_{C}$ & $242991.9^{A}$ & $135881.5 \mathrm{C}$ & $187972.7_{B}$ & 48995.2DE & 85318.1 D & $41820.2_{\mathrm{E}}$ & $38368.3_{\mathrm{E}}$ \\
\hline PECS & 0.0198 & 3.34 & 0.76 & $53311.6 \mathrm{AB}$ & $53705.0_{A B}$ & $40203.91_{C}$ & $43258.3_{B C}$ & $64370.2_{A}$ & $57207.8_{A}$ & $43453.1_{\mathrm{BC}}$ & $54027.9 \mathrm{AB}$ \\
\hline PECC & $<0.0001$ & 12.59 & 0.60 & $37181.2 \mathrm{CDE}$ & $44931.5 \mathrm{CD}$ & $23132.4_{E}$ & $25145.4 \mathrm{DE}$ & $102106.2 \mathrm{~A}$ & $66395.8_{B}$ & ${ }^{34882.83} \mathrm{DE}$ & $57127.43_{\mathrm{BC}}$ \\
\hline SERR & 0.9784 & 0.06 & 0.66 & 433829.4 & 415968.7 & 264635.7 & 520062.5 & 515093.7 & 501781.2 & 179940.5 & 205382.3 \\
\hline $\mathrm{BICP}$ & 0.7887 & 0.35 & 0.70 & 105871.8 & 122828.7 & 56833.5 & 59715.9 & 108728.2 & 111886.1 & 38009.0 & 49217.0 \\
\hline TRIC & $<0.0001$ & 10.15 & 0.75 & $82428.2_{B C}$ & $122069.2_{A}$ & $55298.6_{D E}$ & $57742.5_{\mathrm{DE}}$ & $65084.1_{C D}$ & $88442.5_{B C}$ & $42769.5_{E}$ & $39092.4 \mathrm{E}$ \\
\hline LATS & 0.0002 & 6.98 & 0.82 & $115795.9 \mathrm{D}$ & $178830.8_{\mathrm{AB}}$ & 145041.2 $\mathrm{BCD}$ & $164839.8 \mathrm{ABC}$ & $141648.9_{C D}$ & $182728.0_{\mathrm{A}}$ & $80546.0_{E}$ & 74629.7E \\
\hline FDS & 0.3660 & 1.06 & 0.88 & 123455.9 & 162661.6 & 112828.3 & 130273.5 & 100201.0 & 140260.1 & 145344.8 & 160067.5 \\
\hline ED & 0.4892 & 0.81 & 0.63 & 142415.4 & 210065.0 & 174280.1 & 193797.2 & 120849.6 & 171265.2 & 203983.0 & 212178.3 \\
\hline SUPR & $<0.0001$ & 8.45 & 0.78 & $420343.8_{B}$ & $578718.8 \mathrm{~A}$ & $248500.0_{C D}$ & $306500.0_{C}$ & $208768.4^{D}$ & $257843.7_{C D}$ & $120048.1_{E}$ & $123640.9 \mathrm{E}$ \\
\hline INFR & $<0.0001$ & 13.04 & 0.81 & $236236.3_{C}$ & $367281.2 \mathrm{~A}$ & $307468.8_{B}$ & ${ }^{351375.0} \mathrm{AB}$ & $220343.1_{C}$ & $309031.2_{B}$ & $125310.8_{D}$ & 122255.7 D \\
\hline UTRP & $<0.0001$ & 11.01 & 0.83 & $246579.4_{B}$ & $340968.7_{A}$ & $111785.3 \mathrm{D}$ & $142151.0_{\mathrm{D}}$ & $193649.4_{C}$ & $214504.6_{\mathrm{BC}}$ & $69514.2 \mathrm{E}$ & $75706.6_{E}$ \\
\hline MTRP & $<0.0001$ & 13.32 & 0.82 & $349937.5_{B}$ & $498812.5 \mathrm{~A}$ & $262531.2 \mathrm{CD}$ & $313218.8 B_{C}$ & $159712.2 \mathrm{E}$ & $228075.6 \mathrm{D}$ & $108371.3_{\mathrm{EF}}$ & $102127.3_{\mathrm{F}}$ \\
\hline LTRP & $<0.0001$ & 9.61 & 0.81 & $647812.5_{B C}$ & $1005968.7_{A}$ & $847281.2 \mathrm{AB}$ & $981625.0_{\mathrm{A}}$ & ${ }^{534093.7 C}$ & $733656.2_{\mathrm{BC}}$ & $315876.9 \mathrm{D}$ & $214189.3 \mathrm{D}$ \\
\hline
\end{tabular}

Note: Muscles with significant interactions have their rows highlighted grey; post-hoc differences within that muscle are denoted by letters within data cells; values in a row not sharing a letter are significantly different from one another. $\mathrm{ADEL}=$ Anterior deltoid; $\mathrm{MDEL}=$ Middle deltoid; $\mathrm{PDEL}=$ Posterior deltoid $; \mathrm{PECS}=$ Pectoralis major (sternal head);

PECC = Pectoralis major (clavicular head); SERR = Serratus anterior; BICP = Biceps; TRIC = Triceps; LATS = Latissimus dorsi

FDS = Flexor digitorum superficialis; ED $=$ Extensor digitorum; SUPR = Supraspinatus; INFR = Infraspinatus:

UTRP = Upper trapezius; MTRP = Middle trapezius; LTRP = Lower trapezius 


\section{CONCLUSION}

The results of this study demonstrated differences in muscular activation when changing between single or dual vector elastic resistance and exercise selection. While dual vector exercises typically elevated muscular activation, statistical increases within an exercise existed primarily in abduction and flexion. Due to the increased activity in the scapular stabilizers, shoulder extensors, and horizontal abductors, these exercises may be suitable for individuals rehabilitating a shoulder injury. While improving shoulder muscle activation in flexion and abduction, the addition of the dual vector could allow for greater impacts in the trapezius that are not seen with a single vector. Adding a second vector to our internal or external rotation exercise appeared to provide minimal muscle activity changes.
These findings can be leveraged in upper extremity rehabilitation programs to increase activation in the upper extremity but use of a dual vector is not universally beneficial across exercises. Consideration of the rehabilitation plan, targeted muscles and exercises of interest are needed, as dual vector exercises may not be universally beneficial.

\section{CONFLICT OF INTEREST STATEMENT}

None of the authors have any conflict of interest related to the equipment, methods or results used in this work.

Submitted: April 09, 2020 CDT, Accepted: October 20, 2020

CDT 


\section{REFERENCES}

1. Walton J, Paxinos A, Tzannes A, Callanan M, Hayes $\mathrm{K}$, Murrell G. The unstable shoulder in the adolescent athlete. Am J Sports Med. 2002;30(5):758-767.

2. Sousa N, Mendes R, Monteiro G, Abrantes C. Progressive resistance strength training and the related injuries in older adults: the susceptibility of the shoulder. Aging Clin Exp Res. 2014;26(3):235-240.

3. Escalante G. Exercise modification strategies to prevent and train around shoulder pain. Strength Cond J. 2017;39(3):74-86.

4. Warner J, Caborn D. Overview of shoulder instability. Crit Rev Phys Rehabil Med. 1992;4:145-198.

5. Gomoll A, Hatch G, Millett P. Shoulder injuries in throwing athletes. In: Sports Medicine. ; 2006:200-213.

6. Seroyer S, Nho S, Bach B, Bush-Joseph C, Nicholson $\mathrm{G}$, Romeo A. The kinetic chain in overhand pitching: its potential role for performance enhancement and injury prevention. Sports Health. 2010;2(2):135-146.

\section{Page P, Ellenbecker T. The Scientific and Clinical} Application of Elastic Resistance. Champaign, IL, USA: Human Kinetics; 2003.

8. Hughes C, Hurd K, Jones A, Sprigle S. Resistance properties of Thera-Band tubing during shoulder abduction exercise. J Orthop Sport Phys Ther. 1999;29(7):413-420.

9. Patterson R, Stegink Jansen C, Hogan H, Nassif M. Material properties of Thera-Band tubing. Phys Ther. 2001;81(8):1437-1445.

10. Mikesky A, Topp R, Wigglesworth J, Harsha D, Edwards J. Efficacy of a home-based training program for older adults using elastic tubing. Eur J Appl Physiol Occup Physiol. 1994;69(4):316-320.

11. Simoneau G, Bereda S, Dennis C, Starsky A. Biomechanics of elastic resistance in therapeutic exercise programs. J Orthop Sport Phys Ther. 2001;31(1):16-24.

12. Bang M, Deyle G. Comparison of supervised exercises with and without manual physical therapy for patients with shoulder impingement syndrome. $J$ Orthop Sport Phys Ther. 2000;30:126-137.
13. Page P, Lamberth J, Abadie B, Boling R, Collins R, Linton R. Posterior rotator cuff strengthening using theraband in a functional diagonal pattern in collegiate baseball pitchers. J Athl Train. 1993;28(4):348-350.

14. Batalha N, Dias S, Marinho D, Parraca J. The effectiveness of land and water based resistance training on shoulder rotator cuff strength and balance of youth swimmers. J Hum Kinet. 2018;62:91-102.

15. Treiber F, Lott J, Duncan J, Slavens G, Davis H. Effects of theraband and lightweight dumbbell training on shoulder rotation torque and serve performance in college tennis players. Am J Sports Med. 1998;26(4):510-515.

16. Andersen V, Fimland M, Cumming K, Vraalsen O, Saeterbakken A. Explosive resistance training using elastic bands in young female team handball players. Sport Med Int Open. 2018;2(6):E171-E178.

17. Moseley Jr J, Jobe F, Pink M, Perry J, Tibone J. EMG analysis of the scapular muscles during a shoulder rehabilitation program. Am J Sports Med. 1992;20(2):128-134.

18. AS R, Mohr K, Pink M, Jobe F. Electromyographic analysis of the deltoid and rotator cuff muscles in persons with subacromial impingement. J Shoulder Elb Surg. 2000;9(6):519-523.

19. Oyama S, Myers J, Wassinger C, Lephart S. Threedimensional scapular and clavicular kinematics and scapular muscle activity during retraction exercises. $J$ Orthop Sport Phys Ther. 2010;40(3):169-179.

20. Reinold M, Wilk K, Fleisig G, et al. Electromyographic analysis of the rotator cuff and deltoid musculature during common shoulder external rotation exercises. J Orthop Sport Phys Ther. 2004;34(7):385-394.

21. Joseph R, Alenabi T, Lulic T, Dickerson C. Activation of Supraspinatus and Infraspinatus Partitions and Periscapular Musculature During Rehabilitative Elastic Resistance Exercises. Am J Phys Med Rehabil. 2019;98(5):407-415.

22. Hintermeister R, Lange G, Schultheis J, Bey M, Hawkins R. Electromyographic activity and applied load during shoulder rehabilitation exercises using elastic resistance. Am J Sports Med. 1998;26(2):210-220. 
23. McCann P, Wootten M, Kadaba M, Bigliani L. A kinematic and electromyography study of shoulder rehabilitation exercises. Clin Orthop Relat Res. 1993;288:179-188.

24. Mullaney M, Perkinson C, Kremenic I, Tyler T, Orishimo K, Johnson C. EMG of shoulder muscles during reactive isometric elastic resistance exercises. Int J Sport Phyical Ther. 2017;12(3):417-424.

25. Bosch P, Snyder A, Scherr T, Stupczy M. Differences in shoulder muscle activation patterns during proprioceptive neuromuscular facilitation using manual and elastic band exercise. Athl Train Sport Heal Care. 2011;3(2):69-75.

26. Aboodarda S, Page P, Behm D. Muscle activation comparisons between elastic and isoinertial resistance: A meta-analysis. Clin Biomech. 2016;39:52-61.

27. Calatayud J, Borreani S, Colado J, Martin F, Rogers M. Muscle activity levels in upper-body push exercises with different loads and stability conditions. Phys Sportsmed. 2014;42(4):106-119.

28. Calatayud J, Borreani S, Colado J, Martin F, Tella $\mathrm{V}$, Andersen L. Bench press and push-up at comparable levels of muscle activity results in similar strength gains. J Strength Cond Res. 2015;29(1):246-253.

29. Page P, Topp R, Maloney P, Jaeger E, Labbe A, Stewart G. A comparison of resistive torque generated by elastic resistance and isotonic resistance. In: Journal of Orthopaedic \& Sports Physical Therapy Combined Sections Meeting. ; 2017:A203.

30. Criswell E. Cram's Introduction to Surface Electromyography, Second Edition. Sudbury, ON: Jones \& Bartlett Learning; 2011.

31. Chaffin D. Ergonomics guide for the assessment of human static strength. Am Ind Hygeine Assoc J. 1975;36(7):505-511.

32. McNair PJ, Depledge J, Brettkelly M, Stanley SN. Verbal encouragement: effects on maximum effort voluntary muscle action. $\mathrm{Br}$ J Sports Med. 1996;30(3):243-245.

33. Colado J, Garcia-Masso X, Triplett N, et al. Construct and concurrent validation of a new resistance intensity scale for exercise with TheraBand elastic bands. J Sports Sci Med. 2014;13(4):758-766.
34. Drake J, Callaghan J. Elimination of electrocardiogram contamination from electromyogram signals: an evaluation of currently used removal techniques. J Electromyogr Kinesiol. 2006;16:175-187.

35. Boettcher C, Ginn K, Cathers I. Standard maximum isometric voluntary contraction tests for normalizing shoulder muscle EMG. J Orthop Res. 2008;26(12):1591-1597.

36. Cudlip A, Dickerson C. Regional activation of anterior and posterior supraspinatus differs by plane of elevation, hand load and elevation angle. $J$ Electromyogr Kinesiol. 2018;43(June):14-20.

37. Reinold M, Wilk K, Fleisig G, et al. Electromyographic analysis of the rotator cuff and deltoid musculature during common shoulder external rotation exercises. J Orthop Sport Phys Ther. 2004;34(7):385-394.

38. Copay A, Subach B, Glassman S, Polly D, Schuler T. Understanding the minimum clinically important difference: a review of concepts and methods. Spine J. 2007;7:541-546.

39. Page P. Beyond statistical significance: clinical interpretation of rehabilitation research literature. Int J Sports Phys Ther. 2014;9(5):726-736.

40. Leong H, Tsui S, Ying M, Leung V, Fu S. Ultrasound measurements on acromio-humeral distance and supraspinatus tendon thickness: testretest reliability and correlations with shoulder rotational strengths. J Sci Med Sport.

2012;15:284-291.

41. McCresh K, Crotty J, Lewis J. Acromiohumeral distance measurement in rotator cuff tendinopathy: is there a reliable, clinically applicable method? A systematic review. Br J Sports Med. 2015;49:298-305.

42. Leong H, Tsui S, Ng G, Fu S. Reduction of the subacromial space in athletes with and without rotator cuff tendinopathy and its association with the strength of scapular muscles. J Sci Med Sport. 2016;19:970-974.

43. Leong H, Ng G, Chan S, Fu S. Rotator cuff tendinopathy alters the muscle activity onset and kinematics of scapula. J Electromyogr Kinesiol. 2017;35:40-46.

44. Overbeek C, Kolk A, Nagels J, et al. Increased cocontraction of arm adductors is associated with a favorable course in subacromial pain syndrome. $J$ Shoulder Elb Surg. 2018;27(11):1925-1931. 
45. Charbonnier C, Lädermann A, Kevelham B, Chagué S, Hoffmeyer P, Holzer N. Shoulder strengthening exercises adapted to specific shoulder pathologies can be selected using new simulation techniques: a pilot study. Int J Comput Assist Radiol Surg. 2018;13(2):321-330.

46. Tsuruike M, Ellenbecker T, Lauffenburger C. The application of double elastic band exercise in the 90/ 90 arm position for overhead athletes. Sports Health. 2020;12(5):495-500.
47. Kibler W, Sciascia A. Evaluation and management of scapular dyskinesis in overhead athletes. Curr Rev Musculoskelet Med. 2019;12(4):515-526.

48. Tsuruike M, Ellenbecker T, Nishime R.

Electromyographic analysis of the scapular dyskinesis test in a baseball pitcher with a SLAP lesion: a case report. Int J Sport Phyical Ther. 2020;15:471-477. 